\title{
Risk Scenes Of Managerial Decision-Making With Incomplete Information: An Assessment In Forecasting Models Based On Statistical And Neural Networks Approach
}

\author{
Dusan Marcek ${ }^{1,2}$ \\ ${ }^{1}$ Research Institute of the IT4I Centre of Excellence, Faculty of Philosophy and Science, Silesian University in Opava, \\ Bezruc. Square 13, 74601 Opava, Czech Republic \\ E-mail: dusan.marcek@fpf.slu.cz \\ ${ }^{2}$ The Faculty of Management Science and Informatics, University of Zilina, Slovakia \\ E-mail:dusan.marcek@fri.uniza.sk
}

\begin{abstract}
The paper is concerned with measuring of risks in managerial decision-making. It builds upon the uncertainty of economic information, which is converted into the concept of risk expressed in terms of probability and using confidence intervals and standard deviations of the predicted quantities. The paper explains the relation of a degree of risk expressed by the classical information measure, bit, by the concept of confidence intervals, or possibly by the standard deviation. Forecasting models are applied which are based on a statistical theory and a neural approach. The aim is also to examine whether potentially highly non-linear neural network models outperforms the advanced statistical methods and better reduce risk in managerial decision-making, or they yield competitive results. A method for finding the forecasting horizon within which the risk is minimal is also presented.
\end{abstract}

Keywords: Confidence interval; Entropy; Prediction models; Neural networks; ARIMA/ARCH models; Managerial decision; Risk assessment

\section{Introduction}

An important sphere of information necessary for management of economic processes on all managerial levels is the information about the future development of quantities expressed quantitatively, which is used to characterize the state and the development of the object or process. Evidence shows that it is possible to reduce uncertainty by a suitable choice and use of forecasting models based on statistical methods, soft computing and artificial intelligence methods. In comparison with the manager's expert estimates, these models based on statistical and soft computing methods or artificial intelligence methods are capable of providing information in the form of forecasts of quantities with an acceptable degree of uncertainty. The manager using these forecasts is able to make better decisions, i.e. such decisions whose risks in achieving targets are minimized.

Mathematical statistics Cox and Hinkley and Weisberg (see Refs. 1, 2) offer the theory of point estimates and confidence intervals. The manager can set and influence the span of these confidence intervals. The confidence interval indicates the span of possible values into which falls the future estimate of the forecasted quantity with the chosen probability defined by the manager. This way the limits of the possible future values are set. Point or interval estimates of the future values of various economic indicators are important for the strategic manager's decision-making. When determining information entropy in decision-making, it is useful to focus on how the confidence interval for the forecasted economic quantity can be made more precise, i.e. narrowed by using the forecasting model. A significant prerequisite for the application of such a model in management is that apart from the increased reliability of decision-making, the model output results in uncertainty reduction, which makes decision-making easier and less weighted with risk. The fact or statement that uncertainty reduction facilitates the manager's decision-making is not sufficient. The crucial factor is how specifically the entropy change manifests itself in the consequences of the decision. Not only will it be "easier" to make the decision, but more importantly the decision will be more effective in the long run. 
One of the approaches to understanding uncertainty in forecasting models is understanding it as the standard deviation $\sigma$ of the forecasted quantity used by Marcek (see Refs. 3, 4). This approach is used with measuring risks of prognoses of economic time series and of many economic and financial models and methods. E. g. Applebaum, Bertoin (see Refs. 5, 6) used it in Lévy processes, Havlicky; Medova and Kriacou (see Refs. 7, 8 ) in methods based on the extreme value theory. The standard deviation as a degree of uncertainty or risk of forecasted quantity values estimates is proportional to the statistical degree of accuracy of the forecast defined as Root Mean Square Error (RMSE). This approach is used with measuring risks of prognoses of many economic and financial forecasting models, and in forecasting models of economic time series, models for managing financial risk.

For management, the approach based on the statistical analysis of the dispersion of the quantity values, or on the standard deviation analysis, is the most comprehensible way of representing uncertainty. It is needed to be stated that the standard deviation does not reflect entropy in its true substance as uncertainty which is indicated in bits (binary digits). On the other hand, uncertainty is closely related to how precise are the estimates of the future values of quantities that managers have at their disposal. The less precise the estimate, the larger the standard deviation, and the higher the uncertainty that the information is weighted with. This view of uncertainty does not articulate it in its true sense, however, it expresses very well its inner essence and the mutual relation of entropy and decision-making.

The tasks dealing with the influence of information entropy on managerial decision-making have not been described in literature yet. An important prerequisite is whether the calculation of the degree of uncertainty itself has a sufficient informative value for managers in order for them to follow it during the process of decisionmaking. If it were only a very abstract value insufficiently explained and vaguely described in literature, it would hardly be applied in the process of managerial decision-making, where the data serving as the basis for managerial decision-making must be relevant and clearly presented. Due to the possibility of achieving effects by applying forecasting models in managerial decision-making, an exceptionally interesting, topical and supporting tool offers itself. Our motivation for elaborating it in theory was the fact that to this day there is no theory that would describe the way to reduce or possibly remove uncertainty in management and to use it to calculate effects.

At present, entropy as a measure of uncertainty is described in several publications. The entropy, alongside with two of its derivatives, the relative entropy and the mutual information, have been used with great success in the information-theoretic analysis of communication systems. Our recent research was concerned with the elaboration of the missing theory of entropy as a category of uncertainty and risk in managerial decision-making. Decision-making is one of the basic human activities, whose quality influences the result of the subsequent activities.

The objective of the paper is to point out specific outlines of entropy and risk categories, determine their content in structured decision-making as a category which conditions the ways and methods of management not only at the stage of decision-making but also the impacts at the stage of implementation of the decision, where the consequences of these decisions (effects or losses) will manifest themselves.

The issue of measuring risk in management and its accompanying phenomena is divided into six chapters in the present paper. Chapter two is devoted to characterizing risk and its manifestation in decisionmaking in uncertainty conditions. In the third chapter we present some statistical measures of uncertainty to probe the risk scenes of forecasting models for financial/economic time series. Chapter four is devoted to briefly characterizing two latest modelling methodologies used for high financial time series. In the fifth chapter, risk reduction with the use of forecasting models based on the classical (statistical) methods and models based on artificial intelligence is documented and assessed. Chapter sixth summarizes the main topics results.

\section{Risk and its manifestation in decision-making in uncertainty conditions}

Decision-making has a dominant position in management. Implementation of planning, organization, coordination and work with people and the results of these activities are based on decision-making. Managerial decision-making is understood as the reaction of the manager to the incurred problems, i.e. it is a process of analyzing and thinking, the result of which is a decision. There are many approaches to decision-making. 
Selecting one of them depends on the character of the problem, on the time available, and on the manager's abilities. Herbert Simon (see Refs. 9, 10) distinguishes two ways of decision-making according to the occurrence of the problem at hand: Programmed decision-making deals with problems that the manager has already dealt with before. These are routine and recurring problems. The process of solving these problems is well-established, it is usually possible to convert in into an algorithm, it is often programmed, and standard procedures are usually used. Non-programmed decision-making deals with problems that the manager has not dealt with before. These are more complex and unique problems. The manager does not know in advance how to proceed, and a creative solution is required of him.

Decision-making on the level of lower management usually involves theories and tools such as linear and non-linear programming, dynamic programming, game theory, queuing theory, inventory theory, probability theory, renewal theory, graph theory etc. Decision making on the level of top management is significantly influenced by time. Top management uses tools not only from management but also from other science branches such as mathematical statistics, fuzzy set theory, econometrics, operational research, etc. Top managers use these tools to obtain the most precise estimates of the future development of quantities and processes possible. These estimates represent important information on which managers base their decisions.

Specific choice of tools and models for decisionmaking depends on whether the manager has precise and complete or imprecise and incomplete information at their disposal. The complexity of managerial decisionmaking relates to decision-making with incomplete information. Most of the real systems can only be described incompletely, i.e. with information which cannot be formally expressed by unequivocally set parameters. This is uncertain information then. In practice, according to Ref. 11, there are mainly two types of such information: According to the first type, uncertain information makes it impossible to exactly determine the future behaviour of the examined system. This type of uncertainty is called stochastic, and it can usually be modelled using the probability theory.

Stochastic uncertainty is concerned with the category of the probability risk, which is determined as a scene in the future associated with the specific adverse incident that we are able to predict it using probability theory and a lot of data. In this manuscript, we will concern with this type models, which may be described by Huang (see Ref. 12 ) as follows. Let $D$ be a managerial prediction system including explanatory variables $V$ to explain the behaviour of the variable to be forecast, and faults represented as forecast errors $e_{t}$ in time $t=1,2, \ldots n$. A risk function $R$ in term of the conceptual model $D$ for having a risk scene can be represented as

$$
R=D\left(V, e_{t}\right), \quad t=1,2, \ldots n
$$

To assess the managerial prediction risk $R$ we apply different forecasting models which parameters are estimated by statistical tools.

The second type of uncertainty is connected with the description or formulation of the actual meaning of the phenomena or statements about them. This is semantic uncertainty. Natural language words semantics with uncertainty, i.e. with meanings of words and individual statements not being exact, is typical of natural language. This uncertainty has the character of possibility rather than probability, and it is most often modelled by fuzzy systems. For more details see Refs. 13 - 16. As far as decision-making with risk is concerned, this is the case of decision-making where actual information about real systems is uncertain, and it is not important if the uncertainty is caused by incomplete information about the system's behaviour, or if it is semantic uncertainty. In the further text, in accordance with Ref. 12, the risk connected with managerial decision-making will be modelled using probability models and understood as a statistical term of the expected value between two extreme states of decision, i.e. with full uncertainty and decision with certainty.

\section{Statistical measures of uncertainty}

We present some statistical measures of uncertainty to probe the risk scenes of forecasting models for financial time series. The fact that the classical forecasting models are based on the probability theory makes it obvious that the models are affected by stochastic uncertainty. It is natural that mangers try to obtain maximum utilizable information, i.e. the most precise values of the future estimate possible. For the assessment of the estimate uncertainty degree, the method of confidence intervals for point forecasts can be used.

With determining forecast confidence intervals based on the classical statistical forecasting models such as the models of regression analysis, exponential smoothing, 
and Winter's seasonal models is concerned e.g. by Gaynor and Kirkpatrick (see Ref. 17). A more complicated situation is in case of models based on artificial intelligence such as GMDH or the classical neural networks with adaptation of parameters by the gradient method using Back-propagation algorithm. In this case it is possible to test the $\mathrm{H}_{0}$ hypothesis of the expected type of probability distribution to determine confidence intervals provided that residuals have a normal probability distribution according to Ref. 18 , and this hypothesis can be verified using $\chi^{2}$ test of good fit on levels of significance set in advance. It is a well-known, widely used and relatively universal method of mathematical statistics. To obtain the correct result, it is necessary for the statistical data file to have at least 50 values. The span of the confidence interval is related to the estimate precision. The more precise the input from the forecasting model, the more precisely it is possible to set the span of the confidence interval, and the larger part of uncertainty is removed. Using the $\chi^{2}$ test of good fit, the $\mathrm{H}_{0}$ hypothesis must be verified on the level of significance $\alpha=0.05$ and $\alpha=0.01$, and this hypothesis claims that the residuals of the forecasted values from the actual values can be considered as a data file with a normal probability distribution. The confidence interval can be then calculated according to the following expression

$$
x \in\left\langle\bar{x}-k_{\alpha} \cdot \frac{\sigma}{\sqrt{n}}, \bar{x}+k_{\alpha} \cdot \frac{\sigma}{\sqrt{n}}\right\rangle
$$

where $k_{\alpha}$ is the critical value of the standardized normal probability distribution, $\alpha$ is the level of significance, $\sigma$ is the standard deviation, $n$ is the number of observations, and $\bar{x}$ is the expected value. For the chosen probability $P=0.95$, the confidence interval determines the limits which the estimate value will not exceed with $95 \%$ probability. The value $\alpha=1-P=0.05$ is the so-called level of significance, which means the probability that a random variable of the estimate will acquire a value outside the interval

$$
\left\langle\bar{x}-k_{\alpha} \frac{\sigma}{\sqrt{n}}, \bar{x}+k_{\alpha} \frac{\sigma}{\sqrt{n}}\right\rangle
$$

Interesting about the support of the preference of forecasting models based e.g. on neural networks to managers' expert estimates in managerial decisionmaking is the information about the probability change. The calculation of this probability is possible from expression (1) as the level of significance $k$

$$
k=\bar{x}-a \frac{\sqrt{n-1}}{\sigma_{\text {est }}}
$$

where $\alpha$ is the lower limit of the forecast interval of the prognosis calculated by neural network, $\sigma_{e s t}$ is the standard deviation and $\bar{x}$ is the expected value (mean).

Another measure of uncertainty used in the theory of information is entropy. The entropy, alongside with two of its derivatives, the relative entropy and the mutual information, have been used with great success in the information-theoretic analysis of communication systems (see Refs. 19, 20 for details). Entropy and also uncertainty is expressed by the amount of information that we get after performing an experiment. For example, if we get a message that an event $A$ has occurred with probability $P(A)$, we also get information $I(A)$ equal $-\log _{2} P(A)$ bit.

In case the event $A$ consists of a finite amount of measured events, i.e. subsets of probabilistic space $\Omega$ while $A_{i} \in A$ for $i=1,2, \ldots n, \Omega=\bigcup_{i=1}^{n} A_{i}$ and $A_{i} \bigcap A_{j}=0$ for $i \neq j$ is valid, then the entropy expressed by Sannon's definition is (see Ref. 20)

$$
H(P)=\sum_{i=1}^{n} I\left(A_{i}\right) \cdot P\left(A_{i}\right)=-\sum_{i=1}^{n} I\left(A_{i}\right) \cdot \log _{2} P\left(A_{i}\right)
$$

In this connection, a very important question is, how will the entropy change if the estimate is more precise? The probability used in the relation for the calculation of entropy is the probability that the estimate value will fall into the narrower $95 \%$ confidence interval.

The most frequently measure of uncertainty and risk used in literature is the standard deviation. As far as relevancy is concerned, it is probably the easiest and, for managerial practice, the most comprehensible way of expressing and quantification of uncertainty. While the entropy indicated in the information unit bit is at present a still relatively abstract and almost non-used measure for expressing risk in the sphere of managerial decisionmaking. Uncertainty in the sense of the standard deviation has a higher informative value for managers. Uncertainty expressed by the standard deviation has one drawback, which is unit incompatibility. Entropy is indicated in bits. Despite this fact, as we could see in the given examples, it is easier to work with entropy as the standard deviation. It is possible to state that reduction of entropy of the forecast system was achieved when its standard deviation of forecast errors was reduced. It can be clearly seen in expression (1). In technical systems, rule $3 \sigma$ is used which in the figurative meaning provides 
information about which interval the forecast will almost certainly fall into. Therefore, it provides certainty instead of uncertainty. But it is a certainty which will not push the manager forward with his decision-making if there is a big standard deviation. The real solution leading to the support of decision-making is reducing uncertainty of the forecast system by using a better forecasting model which will achieve lesser variability of prognosis errors. Described in Ref 21, on the basis of prognosis errors analysis, is a method of searching for such a forecast horizon for which entropy and thus also prognosis risk is minimal. A demonstration the procedure of quantifycation of effects arising from the entropy reduction by using different forecasting models can be found in Ref. 22 .

\section{Exchange Rate Forecasting: A Statistical and Neural Approach}

In the remainder of this paper we investigate the application of forecasting models including the two representations based on the statistical/econometric approach and the neural network one respectively to the high frequency forecasting of the exchange rate, the Czech crown (CZK) against the Slovak one (CZK/SKK). We analyse, discuss and compare the forecast accuracy from models which are derived from competing statistical and neural network specifications.

For time series modelling of high frequency data, the statistical regression models with disturbances following ARCH (AutoRegressive Conditional Heteroscedastic) type processes are used. The method of building a statistical appropriate time series forecasting model is an iterative procedure which consists of the implementation of several steps. The main four steps in are: identification, estimation, diagnostic checking, and forecasting. In the iden-tification step a tentative model is identified by studying the behaviour of the autocorrelation function (ACF) and partial autocorrelation (PACF) functions of the Time series. In the estimation step, the estimates for the parameters of the tentative model are computed. In the next step, the diagnostic checks are performed. The Langrange multiplier test is used for testing for heteroscedasticity. The adequacy of the model to the data is checked by testing the significance and relationship of the parameters. To detect non-liner hidden patterns in stochastic financial time series of generally high frequency the fitted residuals are subjected to the BDS test. If any of the tests or residuals is un-acceptable, the model must be re-specified and the previous steps repeated. Once the appropriate model has been found, it can be interpreted and future forecast can be found.

As an alternative to statistical models we use the Radial Basic Function Neural Networks (RBF NN) and their fuzzy logic and granular variants. The neural network in this study was used as a non-linear supplement of the statistical linear ARCH models. The structure of the RBF NN is defined by its architecture (see Fig. 1). In Fig. 1 each circle or node represents the neuron. This neural network consists an input layer with input vector $\mathbf{x}$ and an output layer with the output value $\hat{y}_{t}$.

The output signals of the hidden layer are

$$
o_{j}=\psi_{2}\left(\left\|\mathbf{x}-\mathbf{w}_{j}\right\|\right),
$$

where $x$ is a $k$-dimensional neural input vector, $\mathbf{w}_{j}$ represents the hidden layer weights, $\psi_{2}$ are radial basis (Gaussian) activation functions (RBF) or cloud activation functions (CAF). Note that for an RBF network, the hidden layer weights $\mathbf{w}_{j}$ represent the centres $\mathbf{C}_{j}$ of activation functions $\psi_{2}$.

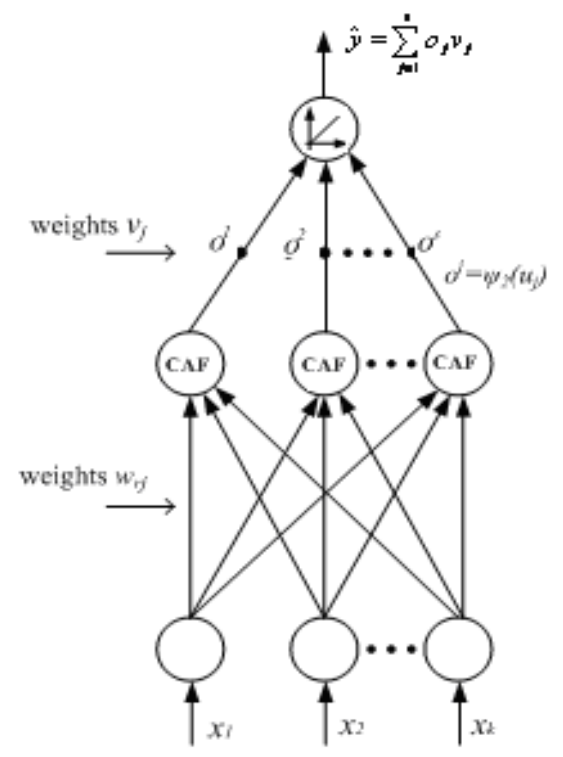

Fig. 1. RBF neural network architecture.

To find the weights $\mathrm{w}_{\mathrm{j}}$ or centres of activation functions we used the adaptive (learning) version of Kmeans clustering algorithm for $s$ clusters. Cluster analysis is regarded as one of the granulation methods, i.e. it say why and how to put objects into same granule. 
Granules are extracted from data in the form of clusters, i.e. these entities receive collections of numerical data that exhibit some functional or descriptive commonalities. The final number of clusters provides the number of granules representing RBF neurons in the hidden layer. The centers of clusters are regarded as the means of granules. A family of granules containing every value of input data is called as a granulated view. The granulated view of input data consists of a family of overlapping granules. The above mentioned learning algorithm based on the clustering is regarded as one of the granular methods presenting bottom-up granulation, i.e. input data are combined into larger granules. The RBF network computes the output data set as

$$
\begin{aligned}
& \hat{y}_{t}=G\left(x_{t}, c, v\right)=\sum_{j=1}^{s} v_{j, t} \psi_{2}\left(x_{t}, c_{j}\right)=\sum_{j=1}^{s} v_{j} o_{j, t}, \\
& t=1,2, \ldots, \mathrm{N}
\end{aligned}
$$

where $N$ is the size of data samples, $s$ denotes the number of the hidden layer neurons (RBF neurons) and $\hat{y}_{t}$ corresponds to the estimated variable $r_{t}$ used in models (4) and (5). According to Ref. (23) the network with one hidden layer and normalised output values $o_{j, t}$, where the normalisation means that the sum of the outputs from the hidden layer is equal to 1 , is the fuzzy logic model or the soft RBF network. In our case, the subjects of learning are the weights $v_{j, t}$ only. These weights can be adapted by the error Back-Propagation algorithm. For detailed computational algorithm for the ex post forecast RMSE values and the weight update rule in the granular network see Ref. 24.

\section{Empirical Studies and Forecasting Performan-ce Evaluation}

As we mentioned above, we will investigate the application of statistical and $\mathrm{RBF} \mathrm{NNs}$ forecasting models on the high frequency time series data of exchange rate CZK/SKK (the Czech crown against the Slovak crown). The data is available at www.oanda.com. The data was collected for the period January 1, 2007 to February 12, 2008 which provided of 702 observations (see Fig. 2). To build a forecast model the sample period (training data set denoted A )for analysis $r_{1}, \ldots, \mathrm{r}_{670}$ was defined, i.e. the period over which the forecasting model was developed and the ex post forecast period (validation data set denoted E ) $r_{671}, \ldots, r_{702}$ as the time period from the first observation after the end of the sample period to the most recent observation. By using only the actual and forecast values within the ex post forecasting period only, the accuracy of the model can be calculated. In our case, the basic unit of time for which the forecasts were made was a day. Economists and financial managers are primarily concerned with short-term forecasting, mostly one month (that is 31 days) in the future. Hence, we chosen the length of validation data set, as the last 31 periods. All 670 previous observations were included into the training data set.

Input selection is crucially important for the successful development of an ARCH-GARCH model. Potential inputs were chosen based on traditional statistical analysis: these included the actual exchange rates $\mathrm{CZK} / \mathrm{SKK}$ and lags thereof.

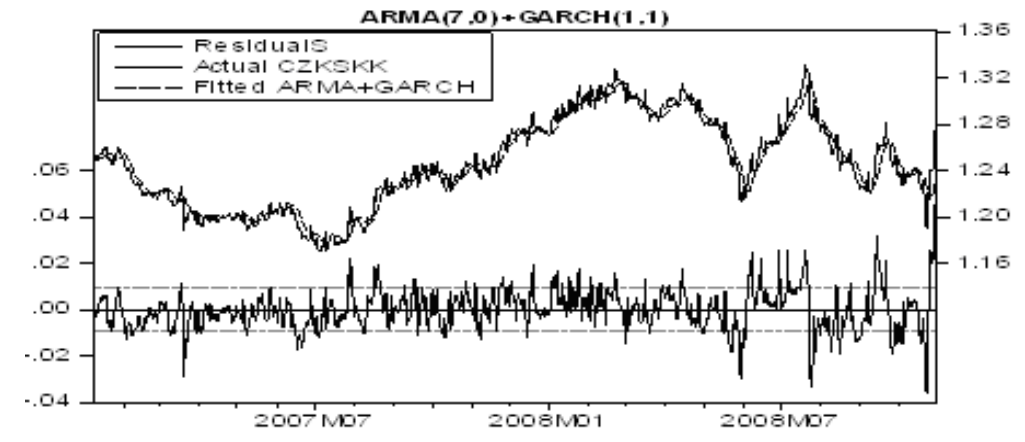

Fig. 2. Actual and fitted values of the CZK/SKK exchange rates-model (6).

Residuals are at the bottom. Actual time series represents the solid line, the fitted values represents the dotted line.
The relevant lag structure of potential inputs was analysed using traditional statistical tools, i.e. using the autocorrelation function (ACF), partial autocorrelation function (PACF) and the Akaike/Bayesian information 
criterion (AIC/BIC): we looked to determine the maximum lag for which the PACF coefficient was statistically significant and the lag given the minimum AIC. According to these criteria the AR(7) model was specified. For estimation the parameters of an $\mathrm{AR}+\mathrm{GARCH}$ model the maximum likelihood procedure was used. The estimation of the model parameters was performed by means of the R-system software and resulted into $\mathrm{AR}(7)+\mathrm{GARCH}(1,1)$ process with mean equation

$$
\begin{aligned}
\hat{r}_{t}= & 0.0012+0.7269 r_{t-1}+0.0929 r_{t-2} \\
& +0.0843 r_{t-3}+0.0821 r_{t-4}-0.0651 r_{t-5} \\
& +0.05591 r_{t-6}+0.0218 r_{t-7}
\end{aligned}
$$

and the variance function

$$
h_{t}=0.0000169+0.3049 e_{t-1}^{2}+0.3616 h_{t-1}
$$

where $e_{t}$ are estimated residuals of $\varepsilon_{t}$ from Eq. (6).

After these findings, we calculated predictions for next 32 trading days These predictions are displayed in Fig. 3.

The same data used for estimating the statistical $\operatorname{AR}(7)+\operatorname{GARCH}(1,1)$ model was used to train the neural network above. The variables forming the right hand site of the Eq. 6 were used as the input units. The results of the ex post forecasts evaluation are shown in Table 1 . According to the RMSE measure neural networks show slightly worse forecasting results than the $\operatorname{AR}(7)+$ $\operatorname{GARCH}(1,1)$ model.

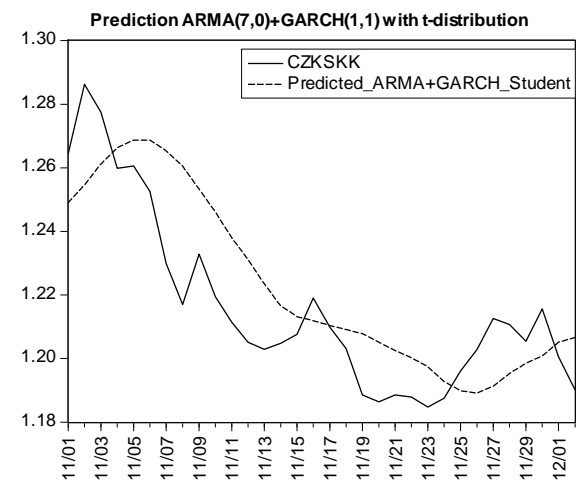

Fig. 3. Actual (solid) and forecast (dotted) values of the $\mathrm{CZK} / \mathrm{SKK}$ exchange rates time series.
Table 1. Ex post forecast RMSEs for ARCH-GARCH model and RBF NNs (see text for details).

\begin{tabular}{lcl}
\hline Model & $\begin{array}{c}\text { Numb. of } \\
\text { RBF } \\
\text { neurons } \\
(s)\end{array}$ & $\begin{array}{l}\text { RMSE } \\
\text { (risk) }\end{array}$ \\
\hline AR(7)+ & & 0.019 \\
GARCH(1,1) & 5 & 0.367 \\
\hline RBF NN(classic) & 10 & 0.367 \\
& 15 & 0.368 \\
\hline RBF NN(soft) & 5 & 0.029 \\
& 10 & 0.030 \\
RBF NN(granular) & 15 & 0.033 \\
\hline
\end{tabular}

Now, the question arises whether there is a method which can help to find an optimal forecasting horizon producing minimal forecast errors. In Ref 25 the exponential smoothing method is described for continually revising of the forecast error by accounting for more recent changes in the data. This method was also applied in Ref. 21 to find the optimal forecasting horizon.

Suppose that we have a history a sequence of forecast errors $e_{1}, e_{2}, \ldots, e_{n}$. We could average all past errors to obtain

$$
\bar{e}_{t}=\sum_{t=1}^{n} e_{t} / n
$$

and to estimate the standard deviation $\hat{\sigma}_{e_{t}}$ of the last $n$ forecast errors as

$$
\hat{\sigma}_{e_{t}}=\sqrt{\frac{\sum_{t=1}^{n}\left(e_{t}-\bar{e}_{t}\right)^{2}}{n-1}}
$$

We logically might wish to give more weight to recent forecast errors than to older data. Hence, we can obtain the smoothed error $S_{t}$ as follows (see Refs. 25, 26)

$$
S_{t}=\alpha e_{t}+(1-\alpha) S_{t-1}
$$

where alpha is the smoothing constant, which falls between zero and one. The smoothing procedure (10) allows us to filter local fluctuations out of our forecast error sequence. This procedure is based on averaging past forecast error values in a decreasing (exponential) manner. The term $S_{t}$ is interpreted as a weighted average. The forecast errors are weighted, with more 
weight given to the more recent forecast errors. The operation defined by Eq. (10) is also called simple exponential smoothing.

Given that we have an estimate of $\hat{\sigma}_{e_{t}}^{2}$, we can estimate the variance of the smoothed forecast errors, $\hat{\sigma}_{s_{t}}^{2}$, as follows

$$
\hat{\sigma}_{S_{t}}^{2}=\frac{\sum_{t=1}^{n}\left(S_{t}-\bar{S}_{t}\right)^{2}}{n-1}
$$

where $\bar{S}_{t}=\sum_{t=1}^{n} S_{t} / n$, and the standard deviation $\hat{\sigma}_{S_{t}}$ of the last $n$ forecast errors as

$$
\hat{\sigma}_{S_{t}}=\sqrt{\frac{\sum_{t=1}^{n}\left(S_{t}-\bar{S}_{t}\right)^{2}}{n-1}}
$$

As an illustration of this concept, consider the analysis of simple exponential smoothing of the fo-recast errors from $\mathrm{AR}(7)+\mathrm{GARCH}(1,1)$ process expressed by equations (5) and (6). Using equations (8) and (9), we can estimate the standard deviations $\hat{\sigma}_{e_{t}}$ for the last forecast errors of validation data set $(t=1,2, \ldots, 31)$ and these one, when simple exponential smoothing for $\alpha=$ $0.05,0.010, \ldots, 0.3$ is used respectively. These values are graphically depicted in Figure 4.

In Figure 4 we can see that from standard deviations of last forecast errors point of view the minimal risk value of our forecasting model can be reached when the forecasting horizon $T$ is lesser than six.

Comparing the risk values of our forecasting model based on MSE's criterion in which each forecast error has the same weights and the method based on exponential smoothing concept, we see that the exponential smoothing concept is better. Both methods can be used for forecast control. When the risk value exceeds the control limit for two or more successive forecasting periods, this may be a strong indication that something is wrong with the forecasting model. E.g. the parameter estimates used in the forecasting model are not accurate and must be improved.

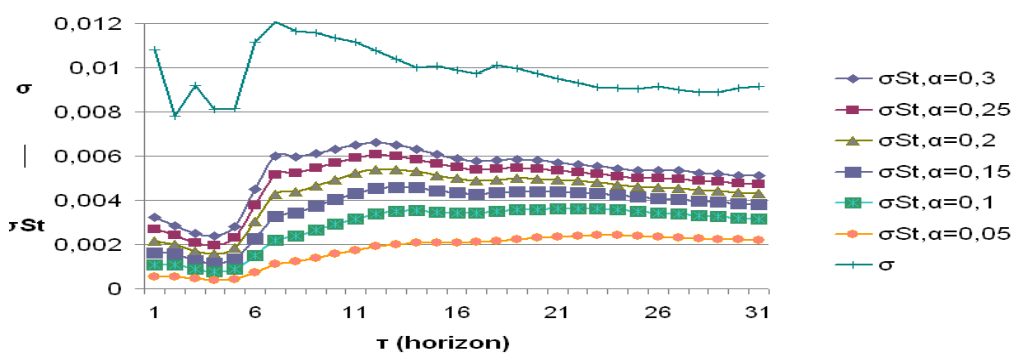

Fig. 4. Estimated values of standard deviations of forecast errors for validation data set and standard deviations, when simple exponential smoothing used.

\section{Conclusion}

In the present paper we showed the procedure of quantitative assessment of risk scene based on probability terms using confidence intervals for point estimates of economic quantities. We build upon measuring uncertainty based on information entropy indicated in bits and on measuring based on prognosis confidence interval, where uncertainty is expressed in terms of the span of the confidence interval and the probability that by using forecasting model the set prognosis limits around the expected value will not be exceeded. We have proposed two approaches for determining the forecast accuracy of the forecasting system applied to exchange rates time series for Czech crown (CZK) against Slovak crown (SKK). The first one was based on the latest statistical ARCH-GARCH methodology, the second one on the RBF NN.

The results of the study showed that there are more ways of approaching the issue of measuring risk in managerial decision-making in companies. It was also proved that it is possible to achieve significant risk reduction in managerial decision-making by applying modern forecasting models based on latest statistical methods and information technologies such as neural networks developed within artificial intelligence. 
In direct comparison between statistical ARCHGARCH models and fuzzy logic and granular RBF NN, the experiment with the daily data indicates that both methodologies yield very little and similar RMSE's values. But our experiment shows that RBF NN models are economical and computational very efficient, well suited for high frequency data forecasting.

We have also shown that applying the exponential smoothing methodology for analysis of past forecast errors in forecasting systems enables as to set an optimal forecasting horizon in which the risk in decision-making process is a minimum.

\section{Acknowledgements}

This paper has been elaborated in the framework of the IT4Innovations Centre of Excellence project, reg. no. CZ.1.05/1.1.00/02.0070 supported by Operational Programme 'Research and Development for Innovations' funded by Structural Funds of the European Union and state budget of the Czech Republic.

\section{References}

1. D. Cox D and D. Hinkley, D. Teoretical Statistics, Chapman and Hall, (London, UK, 1974).

2. S. Weisberg, Applied Linear Regression, Wiley, (New York, USA, 1980).

3. D. Marcek, M. Marcek, J. Babel, Granular RBF NN Approach and Statistical Methods Applied to Modelling and Forecasting High Frequency Data, International Journal of Computational Intelligence Systems, 2-4, (2009):353-64.

4. M. Marcek, Statistical and RBF NN models, Providing forecast and risk assessment, Central European Review of Economic Issues, 12, (2009):175-82.

5. D. Applebaum D. Lévy Processes and Stochastic Calculus, Cambridge, (Cambridge University Press, 2004).

6. J. Bertoin, Lévy Processes of Normal Inverse Gaussian Type, Finance and Statistics, 2, (1998): 41-68.

7. J. Havlicky, Loss distribution approach by applying theory of the extreme values, In Proc. Managing and Modelling of Financial Risk, eds. D. Dluhosova, 4, Czech Republic, Ostrava, ( 2008): 36-41.

8. E. A. Medova, M. N. Kriacou, Extremes in operational risk management, Working Paper, University of Cambridge, (2001).

9. H. A. Simon, Rational Decision Making in Business Organizations, AER, (1979).

10. H. A. Simon, Decision Making and Problem Solving, (1986).
11. V. Olej, Modelling of Economic Processes Based on Computational Intelligence, Pardubice, (The University Press of Pardubice, Czech Republic, 2003).

12. C. F. Huang, A Note on the Difference between Disaster Prediction and Risk Assessment in Natural Disasters, New Perspectives on Risk Analysis and Cisis Response, 9, (Atlantis Press, Paris, France, 2009): 1-7.

13. C. F. Huang, D. Ruan, Fuzzy risks and an updating algorithm with new observation, Risk Analysis, 28 (3): 681-94.

14. S. Ugurlu, C. Kahraman, Fuzzy multicriteria risk assessment for hazardous waste management: The case for Istanbul, Journal of Risk Analysis and Crisis Response, 1(2001): 29-41.

15. N. Mahan, Risk Assessment is Fuzzy Business-Fuzzy Logic Provides the Way to Assess Off-site Risk from Industrial Installations, Risk, 206, (2004).

16. J. H. M. Tah and A. V. Carr, Proposal for construction project risk assessment using fuzzy logic, Construction Management and Economics , 18 (2000) :491-500.

17. P. E. Gaynor, R. C. Kirkpatrick, Introduction to TimeSeries and Forecasting in Business and Economics, (McGraw-Hill, Inc., New York, USA, 1994).

18. A. P. Da Silva AP, L. S. Moulin, Confidence intervals for neural network based short-term load forecasting, Power Systems, IEEE Transactions, 15(4) (2000) :1191-1196.

19. R. M. Gray, Entropy and Information Theory, Springer, New York, (1990).

20. C. E. Shannon. A Mathematical Theory of Communication, Bell System Tech. J., 27 (1948): 379423, 623-656, reprinted in book form with introduction by W. Weaver, (Univ. of Illinois Pres, Urbana, 1949).

21. D. Marcek, M. Marcek, P. Matusik, High Frequency Data: Making Forecasts and Looking for an Optimal Forecasting Horizon, in Proc. of the IEEE 2010 Sixth International Conference on NATURAL COMPUTATION-ICNC 2010, eds. Shigang Yue et al, 4 China, Yantai, Chandong, (2010) : 1686-1691.

22. D. Marcek, M. Frano, and M. Marcek, Managerial decision-making: Measuring and manifestations of risks and the possibilities of their reducing, Journal of Economics, 59(4) (2011): 392-411.

23. V. Kecman, Learning and soft computing: support vector machines, neural networks, and fuzzy logic, (Massachusetts, The MIT Press, 2001).

24. M. Marcek, D. Marcek, Granular RBF Neural Network Implementation of Fuzzy Systems: Application to Time Series Modelling, Journal of Mult.-Valued Logic \& Soft Computing, 14 (2008): 101-14.

25. D. C. Montgomery, L. A. Johnson, J. S. Gardiner, Forecarting and Time Series Analysis, (New York: McGraw-Hill, Inc., 1990). D. Marcek CZK/SKK

26. R. G. Brown, Smoothing, Forecasting, and Prediction of Discrete Time Series, (Englewood Cliffs, PrenticeHall, 1963). 